Abstract

\title{
Toward an Innovative Treatment of Alzheimer's Disease: Design of MTDLs Targeting Acetylcholinesterase and $\alpha-7$ Nicotinic Receptors ${ }^{\dagger}$
}

\author{
Mégane Pons ${ }^{1, *}$, Ludovic Jean ${ }^{1}$, Sylvain Routier ${ }^{2}$, Frédéric Buron ${ }^{2}$, Sylvie Chalon ${ }^{3}$ and \\ Pierre-Yves Renard ${ }^{1}$ \\ 1 Normandie Univ, UNIROUEN, INSA Rouen, CNRS, COBRA (UMR 6014), 76000 Rouen, France \\ 2 ICOA, UMR 7311 CNRS, Université d'Orléans, 45000 Orléans, France \\ 3 UMR 1253, iBrain, Université de Tours, Inserm, 37000 Tours, France \\ * Correspondence: megane.pons@etu.univ-rouen.fr \\ + Presented at the 2nd Molecules Medicinal Chemistry Symposium (MMCS): Facing Novel Challenges in \\ Drug Discovery, Barcelona, Spain, 15-17 May 2019.
}

Published: 27 August 2019

\begin{abstract}
Alzheimer's disease (AD) is a complex and progressive neurodegenerative disorder. The available therapy is limited to symptomatic treatment, and its efficacy remains unsatisfactory. In view of the prevalence and expected increase in the incidence of $\mathrm{AD}$, the development of an effective therapy is crucial for public health. Since the therapeutic paradigm "one compound-one-target" has shown its limits in the treatment of $\mathrm{AD}$, new strategies are emerging to overcome the lack of efficiency of the current pharmacotherapy in the past decade. The most promising is the multitargetdirected ligands (MTDLs) strategy. This project consists of the development of new multifunctional agents, which will act simultaneously on the different players in AD pathology by combining an AChE inhibitory activity based on the structures of a well-known AChE inhibitor (Rivastigmine) with an $\alpha-7 \mathrm{nAChR}$ activation. Indeed, nAChRs were recently put forward as potential targets for the treatment of central nervous system (CNS) diseases, such as AD. Because of their distribution and abundance in the CNS, the $\alpha-7$ subtypes are potential therapeutic targets for this disorder.
\end{abstract}

Keywords: Alzheimer's disease; Multitarget-Directed Ligand; AChE inhibitor; $\alpha-7 \mathrm{nAChR}$ agonist

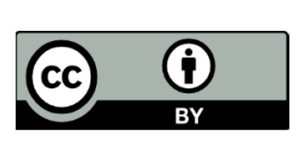

(C) 2019 by the authors. Licensee MDPI, Basel, Switzerland. This article is an open access article distributed under the terms and conditions of the Creative Commons Attribution (CC BY) license (http://creativecommons.org/licenses/by/4.0/). 